\title{
Denovo Epileptiform Encephalopathy in an Elderly Patient as a Result of Psychotrophic Medication Dose Adjustment
}

Hae W Shin ${ }^{1,2^{*}}$ and Tania Beltran Papsdorf

${ }^{1}$ Department of Neurology, University of North Carolina, Chapel Hill, North Carolina, United States

${ }^{2}$ Department of Neurosurgery, University of North Carolina, Chapel Hill, North Carolina, United States

*Corresponding author: Hae W Shin, 170 Manning Drive, CB 7025, University of North Carolina, Chapel Hill, NC 27599, USA, Tel: 919-966-6727; Fax: 919-966-2922; E-mail: shinhw@neurology.unc.edu

Received date: June 16, 2015; Accepted date: August 13, 2015; Published date: August 19, 2015

Copyright: @2015 Shin HW et al. This is an open-access article distributed under the terms of the Creative Commons Attribution License, which permits unrestricted use, distribution, and reproduction in any medium, provided the original author and source are credited.

\begin{abstract}
Psychotropic medications are often used in various psychiatric and neurological conditions. Potential risks of seizures or altered mental status are low but have been reported with these medications. However, increased dose of these medications were not known to predispose to epileptiform encephalopathy. A 79 year old female with a history of mild dementia, major depressive disorder and anxiety was brought into our tertiary medical center by her family for a two week history of worsening depression and anxiety, superimposed on acute psychosis after minor dose adjustment of mirtazapine and donepezil. Neurological evaluation revealed moderate encephalopathy without any underlying metabolic, infectious or structural cerebral abnormalities. However, an electroencephalogram showed frequent bifrontally predominant spikes and polyspikes and slow wave discharges as well as moderate background slowing. Subsequently, an electroencephalogram was normalized after stopping both mirtazapine and donepezil. The patient's mental status was also improved back to her baseline and was able to return home on hospital day 15. To our knowledge, our case is the first report of denovo epileptiform encephalopathy from increased doses of mirtazapine and donepezil which were previously well tolerated in an elderly patient. New onset of psychosis and encephalopathy in these patients should prompt evaluation with EEG.
\end{abstract}

Keywords: Denovo epileptiform encephalopathy in ederly; Psychotropic medications; Mirtazapine; Donepezil

\section{Introduction}

Psychotropic medications are often used in various psychiatric and neurological conditions. Potential risks of seizures or altered mental status and encephalopathy are low but have been reported with these medications [1-5]. Mirtazapine has a risk of seizure of $4.23 \%$ while donepezil has $8.4 \%$ [1-3]. However, increased dose of these medications were not known to predispose to epileptiform encephalopathy. Also, it is unknown that combination of those two medications will have increased risk of seizure or epileptiform encephalopathy. We sought to report denovo epileptiform encephalopathy from increased doses of mirtazapine and donepezil which were previously well tolerated in an elderly patient. We illustrate the effect of psychotropic medication changes on the electroencephalogram (EEG) and correlate this with the effects of withdrawal of the medications both clinically and neurophysiologically.

\section{Methods/Case Description}

A 79 year old female with a history of mild dementia, major depressive disorder and anxiety was brought in by her family for a two week history of worsening depression and anxiety, superimposed on acute psychosis after psychotropic medication changes. The patient had been stable on mirtazapine 30 milligrams (mg) per day and donepezil $5 \mathrm{mg}$ for several months. Due to worsening depression and anxiety, mirtazapine was increased to $45 \mathrm{mg}$ per day and donepezil was increased to $10 \mathrm{mg}$. Due to worsening insomnia with depression, eszopiclone $2 \mathrm{mg}$ was started. Since these changes were made, the patient had acute psychosis with increased confusion, delusions and incoherent speech without any other new medical issues, procedures or changes in diet or home environment. She was admitted to the psychiatric inpatient unit for treatment and evaluation. On admission, eszopiclone was discontinued. Initial metabolic and infectious work up did not reveal any abnormalities. Brain MRI did not reveal any acute abnormalities. Subsequently, neurology was consulted for her encephalopathy on hospital day 3. Her neurological exam showed only moderate encephalopathy.

\section{Result}

A routine electroencephalogram (EEG) was performed with findings of frequent bifrontally predominant spikes and polyspike and slow wave discharges as well as mild to moderate background slowing. Subsequently, inpatient ambulatory EEG was done for 48 hours from hospital day 5 to 7 . Initial findings were similar to the previous routine EEG results (Figure 1). Due to the concern of epileptiform encephalopathy from psychotropic medications, mirtazapine and donepezil were discontinued on the second day of ambulatory monitoring. After discontinuation of these medications, the EEG showed significant improvement with resolution of interictal epileptiform discharges and faster background, up to alpha posterior dominant rhythm (Figure 2). During these two days of monitoring, there were multiple patient events with confusion, agitation, and psychosis without clear electrographic seizures. Over the next several days, her mental status gradually improved. Psychosis resolved and the patient was able to return home on hospital day 15. 
Citation: Shin HW, Papsdorf TB (2015) Denovo Epileptiform Encephalopathy in an Elderly Patient as a Result of Psychotrophic Medication Dose Adjustment. J Neurol Neurophysiol 6: 306. doi:10.4172/2155-9562.1000306

Page 2 of 2

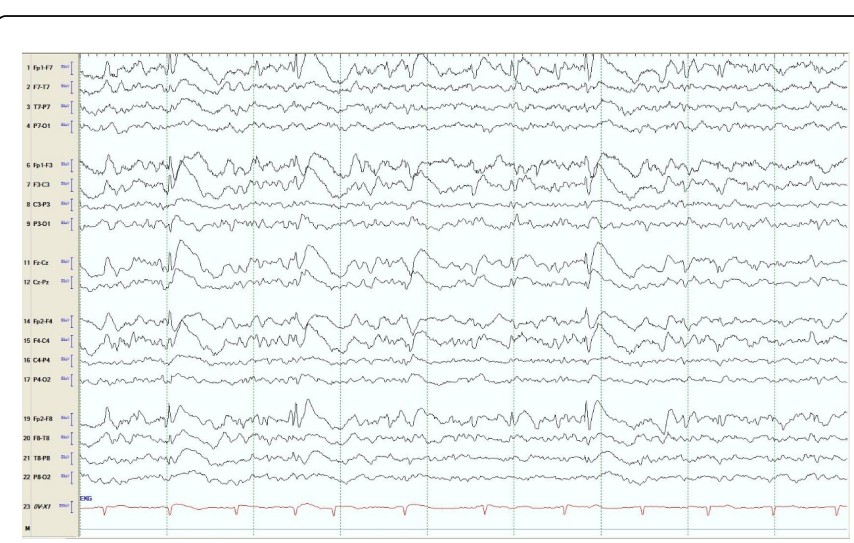

Figure 1: EEG findings of an elderly patient with epileptiform encephalopathy on psychotropic medications. EEG settings: Sensitivity $7 \mathrm{uV}$, High Frequency $70 \mathrm{~Hz}$, Low Frequency $1 \mathrm{~Hz}, 9$ seconds / page.

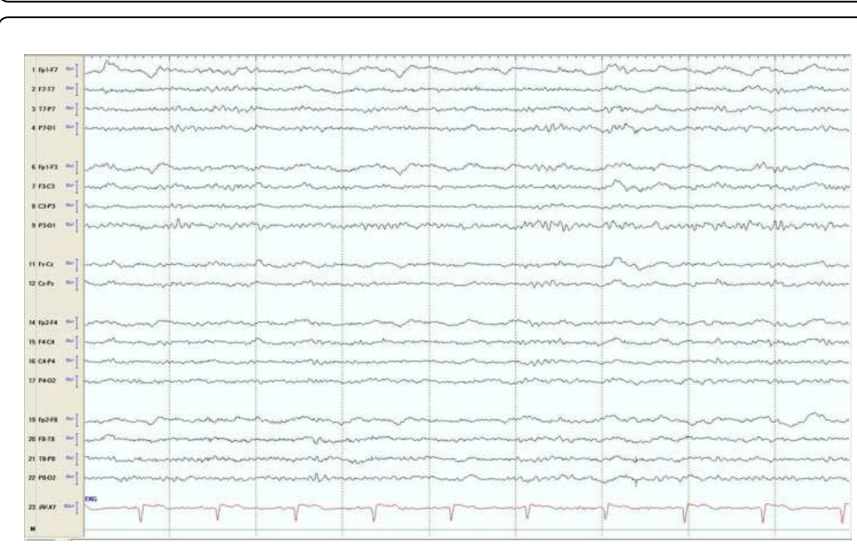

Figure 2: EEG findings of an elderly patient after withdrawal from psychotropic medications. EEG settings: Sensitivity $7 \mathrm{uV}$, High Frequency $70 \mathrm{~Hz}$, Low Frequency $1 \mathrm{~Hz}, 9$ seconds / page.

\section{Discussion}

In general, psychotropic medications are commonly used in various neurological and psychiatric conditions. Most of these medications have relatively safe side effect profile although many of these medications have been reported to cause seizures and encephalopathy. However, even minor changes of psychotropic medications may predispose elderly patients to potential epileptiform encephalopathy and even seizures. To our knowledge, our case is the first report of denovo epileptiform encephalopathy from increased doses of mirtazapine and donepezil which were previously well tolerated in an elderly patient. New onset of psychosis and encephalopathy in these patients should prompt evaluation with EEG.

\section{References}

1. Zia Ul Haq M, Prakash R, Akhtar S (2008) Mirtazapine precipitated seizures: a case report. Prog Neuropsychopharmacol Biol Psychiatry 32: 1076-1078.

2. Spyridi S, Sokolaki S, Nimatoudis J, Iacovides A, Kaprinis G (2009) Status epilepticus in a patient treated with olanzapine and mirtazapine. Int $\mathrm{J}$ Clin Pharmacol Ther 47: 120-123.

3. Kumlien E, Lundberg PO (2010) Seizure risk associated with neuroactive drugs: data from the WHO adverse drug reactions database. Seizure 19: 69-73.

4. Decoutere L, De Winter S, Vander Weyden L, Spriet I, Schrooten M, et al. (2012) A venlafaxine and mirtazapine-induced serotonin syndrome confirmed by de- and re-challenge. Int J Clin Pharm 34: 686-688.

5. Thornton SL, Clark RF (2014) Encephalopathy from unintentional donepezil and memantine ingestion. Pediatr Emerg Care 30: 649-650. 